\title{
Two-Wavelength Square-Waveform Generation Based on Fiber Optical Parametric Oscillator
}

\author{
Sigang Yang, Chi Zhang, Yue Zhou, and Kenneth Kin-Yip Wong, Member, IEEE
}

\begin{abstract}
A square-waveform generator based on a fiber optical parametric oscillator (FOPO) is proposed and demonstrated experimentally. The FOPO works fundamentally by extracting the longitudinal modes beating signal to drive the intracavity amplitude modulator. In order to broaden the longitudinal mode spacing, two sub-ring cavities with a short cavity length are used. The longitudinal modes are phase locked, which ensures the generated microwave signal with very low phase noise. By biasing the amplitude modulator, the even-order beating signals are suppressed, while the odd-order beating signals are enhanced. Thus, the FOPO is self-starting and can provide stable square-waveform output with the repetition rate of $1.133 \mathrm{GHz}$ at the signal and idler simultaneously. It is tunable over $10 \mathrm{~nm}$, which is only limited by the gain bandwidth of the fiber optical parametric amplifier of the current configuration.
\end{abstract}

Index Terms-Microwave photonics, nonlinear optics, optical fiber lasers, optical generation of microwave signal, parametric amplifiers (AMPs), parametric oscillators.

\section{INTRODUCTION}

$\mathbf{O}$ PTICAL generation of microwave signals is an important research branch in microwave photonics [1]-[8]. Usually the techniques to generate microwave signal in the optical domain make use of the beat note of phase correlated optical frequencies [1]. Thus, in order to generate a microwave signal in the optical domain, the primary step is to obtain the phase-locked optical frequencies. Thanks to the state-of-the-art mode-locking techniques [9]-[12], the mode-locked lasers provide an ideal phase-locked frequency source for the beating. Fiber lasers, in which, the active gain medium is an optical fiber doped with rare-earth elements, have attracted more and more attention due to their remarkable advantages such as high power, high stability, high reliability, and so on [13]. For a fiber laser, in order to obtain enough gain so as to overcome the cavity loss, long length of the gain fiber, such as erbium-doped fiber (EDF), is required. A major problem with the long cavity is that the longitudinal mode spacing is fairly small, typically in the order of megahertz or even kilohertz. Hence, it is difficult to make an intracavity optical filter to select and track a single

Manuscript received January 06, 2010; revised July 28, 2010; accepted July 29, 2010. Date of publication September 27, 2010; date of current version November 12, 2010. This work was supported in part by the Research Grants Council of the Hong Kong Special Administrative Region, China, under Grant Project HKU7179/08E and Grant Project HKU7183/09E.

The authors are with the Photonic Systems Research Laboratory, Department of Electrical and Electronic Engineering, The University of Hong Kong, Hong Kong (e-mail: sgyang@eee.hku.hk; zhangchi@eee.hku.hk; yzhou@eee.hku.hk; kywong@eee.hku.hk).

Color versions of one or more of the figures in this paper are available online at http://ieeexplore.ieee.org.

Digital Object Identifier 10.1109/TMTT.2010.2074610 or several frequencies. Consequently, multiple longitudinal modes oscillate simultaneously. Under normal circumstances, phases of these multiple longitudinal modes have random relationships, and for a continuous wave $(\mathrm{CW})$ oscillation beam, intensity shows random time variation, as well as noise [14]. In order to overcome this problem, an actively mode-locked method has been proposed to lock the phase of the longitudinal modes [10]. In the actively mode locked fiber laser, an external RF signal is used to drive the intracavity amplitude modulator. As a result, all the longitudinal modes have the same spectral phases so that they can serve as the beating source in order to produce a microwave signal. For example, a rational harmonic mode-locked fiber ring laser has been proposed to generate a microwave signal [15]. Another optical oscillator is the so-called fiber optical parametric oscillator (FOPO), which utilizes the fiber optical parametric amplification (FOPA) as the gain mechanism instead of the EDF [16]. One advantage of the FOPO over a traditional fiber laser is that it can provide two synchronized output wavelengths at the signal and idler simultaneously. Thus, photonic generation based on the FOPO can provide two-wavelength synchronized microwave signal simultaneously. Hence, the fiber optical parametric amplifiers (AMPs) and oscillators have attracted more and more interests in the study of microwave photonics, as they provide more freedom to tailor the output signal compared with the traditional EDF-based fiber lasers [17], [18]. Basically, the $\mathrm{CW}$ FOPO is the multiple longitudinal mode due to the long cavity length [19], [20]. The longitudinal modes have a random phase relationship. In order to overcome this problem, we have previously proposed an actively mode-locked FOPO to lock the phase of the longitudinal modes [21]. However, in an actively mode-locked fiber laser or FOPO, the RF signal frequency from the external signal generator must be the precise harmonics of the free spectral range (FSR) of the fiber cavity. The cavity easily loses synchronism with the modulation signal because it will readily drift due to the temperature variation or environmental disturbance. As a result, the generated microwave signal is not stable and durable, and it can only provide a single frequency microwave signal based on this method [15].

To synchronize the modulation frequency and the cavity automatically, a regenerative mode-locked FOPO which used an opto-electronic oscillator (OEO) as the modulation signal source was proposed [22]. The main limitation is that the OEO requires a high- $Q$ RF narrowband electrical filter. Alternatively, the RF driving signal in an actively mode-locked FOPO can be directly acquired from the beat note between the multiple longitudinal modes. In this paper, we propose and demonstrate experimentally a novel method for microwave signal generation based on a regeneratively mode-locked FOPO, which simply 
employs the beating RF signal of the longitudinal modes to drive the intracavity amplitude modulator. Due to the long cavity length of the FOPO, we use two sub-rings with short cavity length to enlarge the effective longitudinal mode spacing, as well as increasing the modulation frequency. The FOPO can produce a two-wavelength stable square pulse train with the repetition of $1.133 \mathrm{GHz}$ at the signal and idler without any external RF signal source. The tuning range is over $10 \mathrm{~nm}$, which is limited only by the FOPA gain bandwidth under the current CW FOPO pump configuration. The proposed scheme provides a solution that produces pulse train with potentially higher repetition rates than that of the traditional techniques. The repetition rate can be increased by adopting the following steps. The first is to use the sub-rings with shorter cavity length. This can be realized by decreasing the length of the pigtails of the devices in the sub-rings, e.g., the 50/50 coupler and the polarization controller. The shorter cavity length will result in larger FSR for the individual sub-ring cavity. The second step is to introduce more sub-ring cavities with different cavity length. The resultant least common multiple of the FSRs of all cavities will increase remarkably compared with the current two sub-ring cavities configuration. This also requires higher operating frequency for the amplitude modulator. Hence, the repetition rate of the generated pulse train is limited by the available operating bandwidth of the intracavity amplitude modulator.

\section{PRINCIPLE}

For an optical ring oscillator, the resonant frequencies can be obtained by imposing the condition that the total phase shift along the ring path must equal to an integral number of $2 \pi$. The phase condition can be described by $\beta L=2 k \pi$ [23]-[25] in which $\beta$ is the propagation constant in the ring, $L$ is the cavity length, and $k$ is a positive integer. The spacing between two consecutive longitudinal mode frequencies is

$$
D v=c / n L
$$

where $c$ is the light speed in vacuum, $n$ is the equivalent refractive index of the ring, and $L$ is the cavity length. Under normal circumstances, the multiple longitudinal modes have random phase relationships, as can be observed in a self-homodyne measurement [26], [27]. This measurement acquires the beating signal of the longitudinal modes. The fundamental and higher order beating signals can be observed clearly. However, the beating signals are not stable and have severe fluctuation, which indicates that the longitudinal modes contain severe phase noise. The mode-locking technique is used to lock the spectral phase of the longitudinal modes, thus the spectral phase difference of two consecutive modes is constant. The active mode-locking method is a typical resolution to lock the phase of the longitudinal modes [28]. In an active mode-locked resonant cavity, the AM produces sidebands to each of the oscillating longitudinal modes at the longitudinal mode spacing. The sidebands have a fixed spectral phase difference. Furthermore, the sidebands are modulated with the same frequency by AM and higher order sidebands will be produced, as shown in Fig. 1.

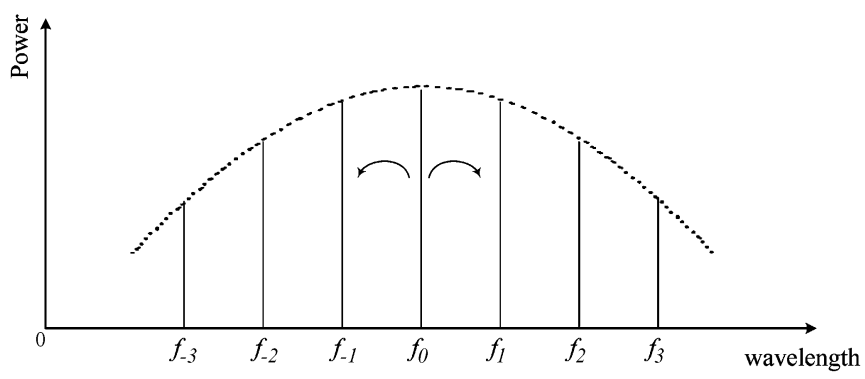

Fig. 1. Principle of the active mode locking.

Finally, the spectral phase $\varphi_{l}$ of the longitudinal modes in the output beam is locked according to the relation

$$
\varphi_{l}-\varphi_{l-1}=\Delta \varphi
$$

where $\Delta \varphi$ is constant. The modulator transfers energy from each mode to its neighboring mode, thereby redistributing energy from the center to the wings of the spectrum. This process seeds and injection locks neighboring modes. Since the longitudinal modes are phase locked in a mode-locked resonant cavity, the microwave signal can be generated based on optical heterodyne in which the multiple longitudinal modes beat at a photodetector (PD). An electrical beat note is generated at the output of the PD with the frequencies at the fundamental and multiple longitudinal mode spacing.

Due to the long cavity length of the fiber resonant cavity, the longitudinal mode spacing is very small, which results in a microwave beating signal with very low frequency. According to (1), the longitudinal mode spacing is the inverse ratio of the cavity length. In order to obtain a high-frequency beat note, a coupled sub-ring cavity with shorter cavity length can be introduced. For a multiple-ring composite cavity, the modes oscillate only at frequencies that satisfy the resonant conditions of the main cavity and all of the sub-ring cavities simultaneously. Therefore, the introduction of the short ring cavities will significantly increase the effective longitudinal mode spacing.

Usually, for an actively mode-locked resonant cavity, an external frequency source is necessary for driving the intracavity amplitude modulator. Thus, the generated microwave signal has the frequency equal or integral multiple of the external signal frequency. The driving signal to the intracavity AM can also be obtained directly from the beat note between the longitudinal modes instead of an external microwave signal source. Particularly, a portion of the oscillating light is extracted and detected by a PD first. While the beat note is noisy and not stable because the longitudinal modes are not phase locked. The generated microwave signal is then amplified and time delayed and fed back to drive the intracavity AM. Although the longitudinal modes have a random phase relationship initially, the oscillating longitudinal modes will be phase locked after such a positive feedback. Different from [15], the generated microwave signal consists of the fundamental and integral multiple of the cavity FSR frequency components rather than a single frequency. By tailoring the electrical spectrum in the frequency domain, the desired waveform in the time domain can be obtained. This can be realized by biasing the intracavity AM at 


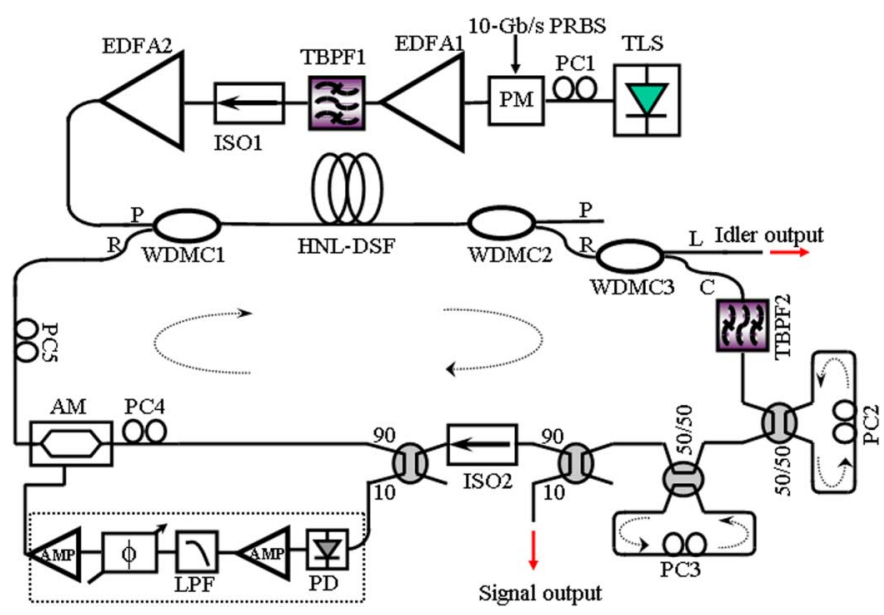

Fig. 2. Schematic diagram of the square-waveform generator based on FOPO. The dotted box corresponds to the electrical path.

a proper voltage. For example, the even-order sidebands can be suppressed, while the odd-order sidebands can be reserved when biasing the Mach-Zehnder AM at the minimum point of the transfer function [1]. In the time domain, the resonant cavity will produce the square waveform.

\section{EXPERIMENT}

The schematic diagram of the square-waveform generator based on FOPO is shown in Fig. 2. The pump was seeded by an external cavity tunable laser source (TLS). In order to suppress the stimulated Brillouin scattering (SBS), the light was first phase modulated with $10-\mathrm{Gb} / \mathrm{s}$ pseudorandom bit sequence (PRBS) signal via a phase modulator (PM). The polarization controller PC1 aligned the state of polarization (SOP) of the pump with the transmission axis of the PM. The SBS could be suppressed by up to $28 \mathrm{~dB}$. The pump was then amplified by a two-stage configuration of EDFAs in which the first stage (EDFA1) provided a small-signal gain to prevent self-saturation by amplified spontaneous emission (ASE). It was then filtered by a $0.8-\mathrm{nm}$ tunable bandpass filter (TBPF1) to reduce ASE noise, and further amplified by the second stage (EDFA2), with a maximum average output power of 33 $\mathrm{dBm}$. The amplified pump was coupled into a 400-m highly nonlinear dispersion-shifted fiber (HNL-DSF) via the $P$-port (1554.89-1563.89 $\mathrm{nm}$ ) of a bandpass wavelength-division multiplexing (WDM) coupler (WDMC1). The high power pump propagated through the HNL-DSF and then was rejected via the $P$-port of another bandpass WDM coupler (WDMC2), while the amplified signal and idler propagated through the $R$-port (1500-1551 nm, 1567-1620 nm) of WDMC2. A $C / L$-band WDM coupler was then inserted to allow only the $C$-band signal to circulate in the cavity, while the idler was coupled out via the $L$-port. The signal from the $C$-port of WDMC3 was filtered by a $0.35-\mathrm{nm}$ bandpass filter (TBPF2), which determined the lasing wavelength. Two sub-ring cavities with different cavity length were then inserted after TBPF2. The FOPO provided the output through 10/90 output coupler. Another $10 / 90$ coupler was used to provide feedback. The feedback loop, as shown in the dashed square region, consisted of a PD
TABLE I

SPECIFICATIONS OF HNL-DSF

\begin{tabular}{lc}
\hline \hline \multicolumn{1}{c}{ Parameter } & Value \\
\hline Length & 400 meters \\
Attenuation & $0.56 \mathrm{~dB} / \mathrm{km}$ \\
Dispersion & $-0.14 \mathrm{ps} / \mathrm{km} / \mathrm{nm} @ 1550 \mathrm{~nm}$ \\
Dispersion slope & $0.035 \mathrm{ps} / \mathrm{km} / \mathrm{nm}^{2} @ 1550 \mathrm{~nm}$ \\
MFD & $3.8 \mu \mathrm{\mu m}$ \\
Nonlinear coefficient & $14 W^{-1} \mathrm{~km}^{-1}$ \\
PMD & $0.03 \mathrm{ps} / \mathrm{sqrt}(\mathrm{km})$ \\
Zero dispersion wavelength & $1554 \mathrm{~nm}$ \\
\end{tabular}

with 12-GHz bandwidth, a 7.5-GHz low-pass filter (LPF), a variable electrical phase shifter denoted by $\phi$, and RF AMPs. A $\mathrm{LiNbO}_{3}$ Mach-Zehnder amplitude modulator (MZ-AM) driven by the feedback signal was inserted. Due to the polarization dependence of the MZ-AM, a polarization controller (PC4) was employed to adjust the SOP of the signal pulse. PC5 was used to align the signal's SOP with that of the pump so as to maximize the signal gain.

The specifications of the HNL-DSF are shown in Table I. Its nonlinear coefficient was $14 \mathrm{~W}^{-1} \mathrm{~km}^{-1}$ and its zero dispersion wavelength was $1554 \mathrm{~nm}$. The OPA worked in its anomalous dispersion region, as the pump wavelength was $1556 \mathrm{~nm}$ [29] The pump phase dithering would not influence the microwave beat note and the output signal [30]. The beat note is only determined by the effective FSR of the composite ring cavities. In order to broaden the effective longitudinal mode spacing, two short sub-ring cavities with different cavity length were introduced. The PC in each sub-ring cavity must be tuned to the same SOP as that of the main cavity. The main cavity and the sub-ring cavities are on resonance. Hence, the radiation can couple effectively from a mode of the main cavity to modes of the sub-ring cavities [31]. The introduction of the sub-ring cavities can suppress the oscillation of longitudinal modes of the main cavity greatly. The cavity lengths of the two sub-ring cavities are about 6.2 and $6.5 \mathrm{~m}$, respectively. The effective FSR of the three cavities was measured using a self-homodyne method and the effective longitudinal mode spacing with all three cavities connected was $1.133 \mathrm{GHz}$. In our scheme, a portion of the optical signal from the main cavity was extracted through a 10/90 coupler and detected by a PD. The generated RF signal from the PD consisted of the fundamental beating frequency and the harmonics. It was amplified by an electrical AMP first. A 7.5-GHz electrical LPF was used to allow only lower order harmonics to be transmitted and suppress high-frequency noise. An electrical phase shifter was used to adjust the phase difference between the circulating pulses and the modulation signal so as to ensure perfect synchronization. After the RF signal was further amplified by a second electrical AMP, the amplified signal was fed back to drive the MZ-AM. The FOPO oscillation was established once the pump power exceeded the threshold power. The oscillation was self-starting and the process was like the following. Initially the longitudinal modes separated equally by $1.133 \mathrm{GHz}$ showed random phase relationship. Thus, the fundamental and higher order beating signal was very noisy and unstable owing to the phase noise. 


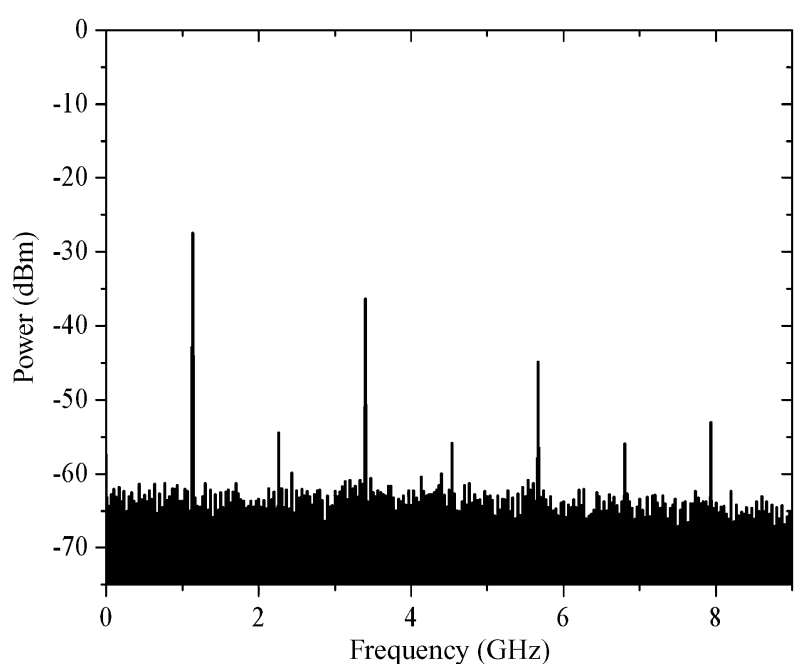

(a)

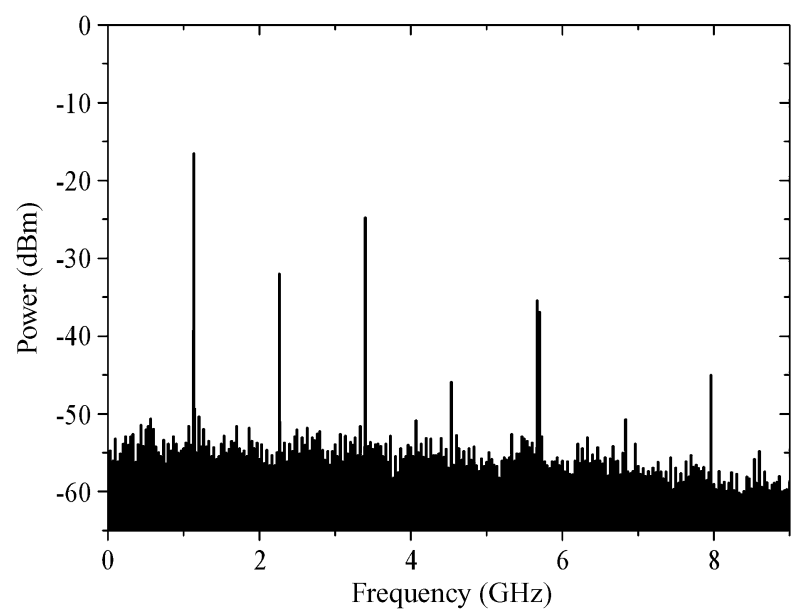

(b)

Fig. 3. Measured RF spectrum. (a) Signal pulse train. (b) Idler pulse train.

However, when the generated RF signal from the PD drove the MZ-AM biased at the voltage of $1.28 \mathrm{~V}$, the MZ-AM produced sidebands to each of the oscillating longitudinal modes. Since the sidebands had fixed spectral phase difference, the longitudinal modes were mode locked. The optical signal output port provided stable pulse train. Fig. 3(a) and (b) shows the measured RF spectrum of the output signal and idler pulse trains, respectively, using an electrical spectrum analyzer (ESA). The fundamental frequency and the odd harmonics can be observed while the even harmonics are prohibited. The reason is that the MZ-AM was biased at the quadrature point at which even harmonics were suppressed [32].

The generated optical pulse trains were observed on a digital communication analyzer (DCA) and are shown in Fig. 4. Fig. 4(a) and (b) shows the signal and idler waveforms, respectively. The FOPO provides square-waveform pulse trains at the signal and idler wavelengths simultaneously. The overshoot is due to the limited bandwidth of the electrical branch in the FOPO. The measured duty cycle is $45.3 \%$ and the repetition rate is $1.133 \mathrm{GHz}$. The simultaneous generation of the signal and idler during the OPA process indicates that each photon pair is correlated in the quantum sense [30]. Thus, the proposed

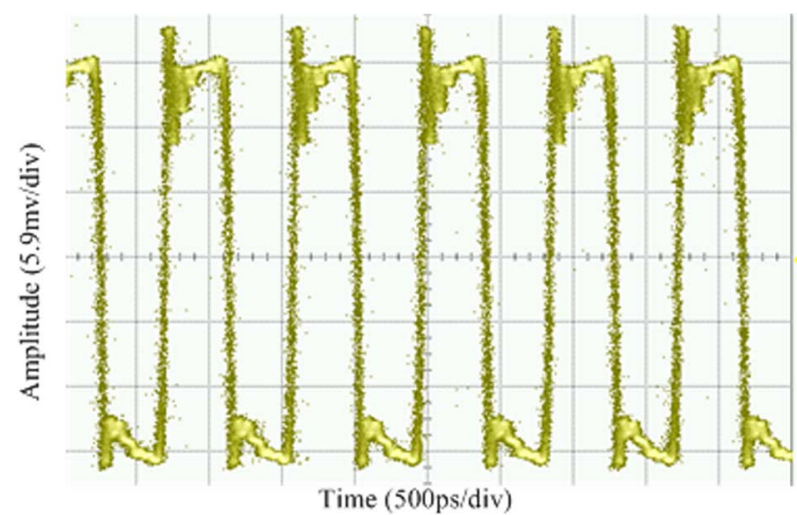

(a)

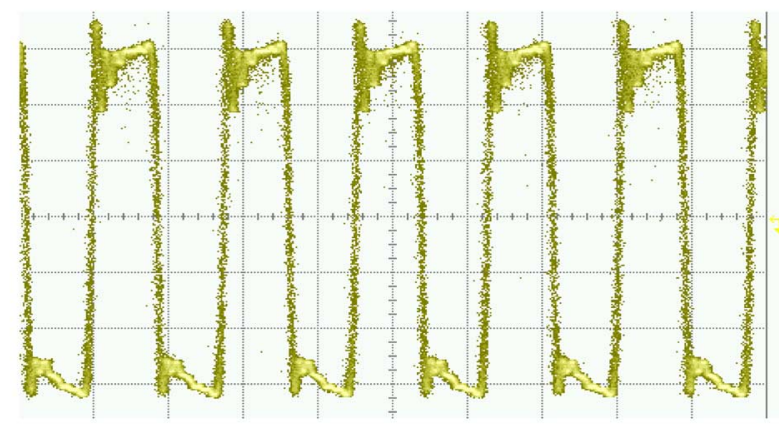

(b)

Fig. 4. Measured waveforms of the output using DCA. (a) Signal. (b) Idler.

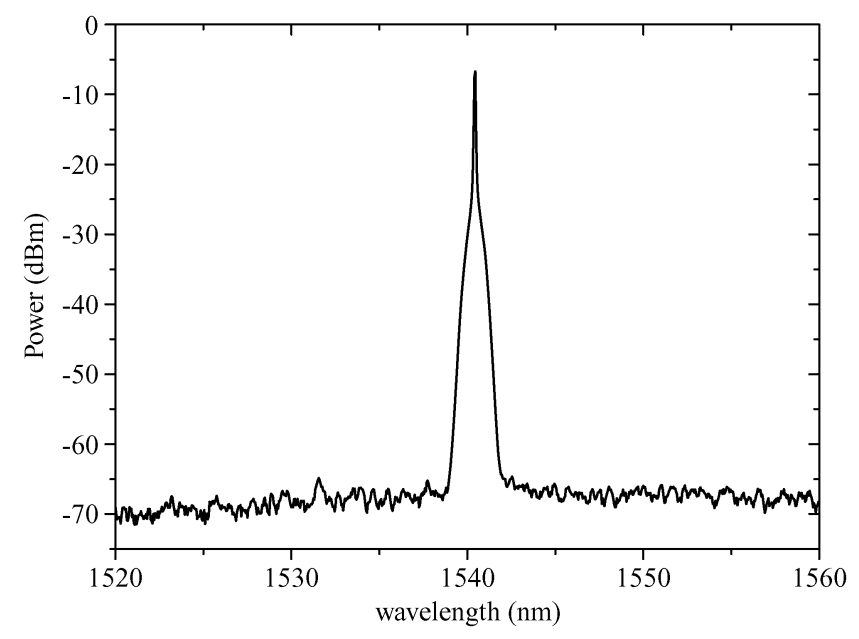

Fig. 5. Measured optical spectrum of the output signal pulse train.

scheme provides an ideal synchronized signal source for the application in modern large-scale scientific facilities requiring the synchronized time signals. The optical spectrum of the pulse train is shown in Fig. 5. The lasing wavelength has more than $60-\mathrm{dB}$ suppression ratio over other nonlasing modes. It indicates that, with the proposed method, the longitudinal modes are well phase locked and the phase noise can be negligible. The FOPO was self-starting and could maintain stable square-waveform pulse train.

The FOPO could also be tunable without disturbing the stable pulsing state. When the oscillating wavelength is varied, the longitudinal mode spacing would change due to the cavity dispersion. In a traditional actively mode-locked FOPO, the MZ-AM 


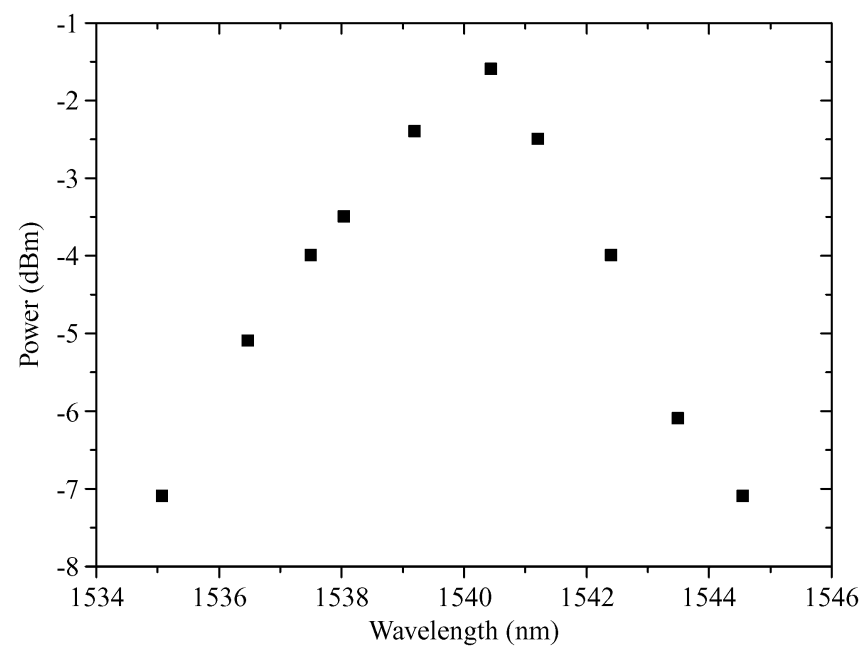

Fig. 6. Output power of the FOPO versus wavelength.

is driven by an external RF signal generator. To maintain the synchronization, the repetition rate of the signal or the cavity length has to be tuned. However, in our scheme, the longitudinal mode beating signal acts as the driving RF signal and it is synchronized with the cavity automatically. For the signal, the maximum output power was $-1.6 \mathrm{dBm}$ at the wavelength of $1540.45 \mathrm{~nm}$ when the pump power was $1.28 \mathrm{~W}$.

However, when the oscillating wavelength was tuned away from $1540.45 \mathrm{~nm}$, the output power would decrease due to the gain bandwidth of the FOPA. Fig. 6 shows the output power versus wavelength when the FOPO was tuned. It shows the FOPO can be tuned over $10 \mathrm{~nm}$. Actually the gain spectrum of the FOPA is not uniform over its gain bandwidth. The reason is that it is hard to maintain the phase-matching condition over a wide bandwidth in a single-pump FOPA. The cavity loss is as large as $20 \mathrm{~dB}$. Only when the OPA gain exceeds the cavity loss, the cavity can oscillate. Thus, there is only a narrow gain bandwidth that can provide such large gain. Hence, it decides the tuning range.

\section{CONCLUSION}

In conclusion, we have demonstrated photonic generation of the square waveform using a FOPO. The FOPO worked fundamentally by extracting the beating signal of the longitudinal modes to drive the intracavity amplitude modulator. In order to broaden the longitudinal mode spacing, two sub-ring cavities with short cavity length were inserted. The resultant longitudinal mode spacing could be broadened to be $1.133 \mathrm{GHz}$. The generated RF signal from the PD drove the intracavity amplitude modulator. Without any external RF signal source, the FOPO could output square-waveform pulse trains with the repetition rate of $1.133 \mathrm{GHz}$ at the signal and the idler wavelengths simultaneously.

\section{ACKNOWLEDGMENT}

The authors would like to acknowledge Sumitomo Electric Industries, Yokohama, Japan, for providing the HNL-DSF.

\section{REFERENCES}

[1] J. P. Yao, "Microwave photonics," J. Lightw. Technol., vol. 27, no. 2 , pp. 314-335, Feb. 2009

[2] L. Goldberg, H. F. Taylor, J. F. Weller, and D. M. Bloom, "Microwave signal generation with injection locked laser diodes," Electron. Lett., vol. 19, no. 13, pp. 491-493, Jun. 1983.

[3] L. Goldberg, A. Yurek, H. F. Taylor, and J. F. Weller, " $35 \mathrm{GHz}$ microwave signal generation with injection locked laser diode," Electron. Lett., vol. 21, no. 18, pp. 714-715, Aug. 1985.

[4] J. J. O'Reilly, P. M. Lane, R. Heidemann, and R. Hofstetter, "Optical generation of very narrow linewidth millimeter wave signals," Electron. Lett., vol. 28, no. 25, pp. 2309-2311, Dec. 1992.

[5] M. Hyodo, M. Tani, S. Matsuura, N. Onodera, and K. Sakai, "Generation of millimeter-wave radiation using a dual-longitudinal-mode microchip laser," Electron. Lett., vol. 32, no. 17, pp. 1589-1591, Aug. 1996.

[6] Z. Fan and M. Dagenais, "Optical generation of a mHz-linewidth microwave signal using semiconductor lasers and a discriminator-aided phase-locked loop," IEEE Trans. Microw. Theory Tech., vol. 45, no. 8, pp. 1296-1300, Aug. 1997.

[7] P. Shen, N. J. Gomes, P. A. Davies, W. P. Shillue, P. G. Huggard, and B. N. Ellison, "High-purity millimeter-wave photonic local oscillator generation and delivery," in Proc. Int. Microw. Photon. Top. Meeting, Sep. 10-12, 2003, pp. 189-192.

[8] G. Qi, J. P. Yao, J. Seregelyi, C. Bélisle, and S. Paquet, "Optical generation and distribution of continuously tunable millimeter-wave signals using an optical phase modulator," J. Lightw. Technol., vol. 23, no. 9, pp. 2687-2695, Sep. 2005.

[9] Z. Li, C. Lou, K. Chan, Y. Li, and Y. Gao, "Theoretical and experimental study of pulse-amplitude-equalization in a rational harmonic mode-locked fiber ring laser," IEEE J. Quantum Electron., vol. 37, no. 1, pp. 33-37, Jan. 2001.

[10] Y. Parkhomenko, M. Horowitz, C. R. Menyuk, and T. F. Carruthers, "Theoretical study of an actively mode-locked fiber laser stabilized by an intracavity Fabry-Perot etalon: Linear regime," J. Opt. Soc. Amer. B, Opt. Phys., vol. 24, pp. 1793-1802, Aug. 2007.

[11] J. Yao, J. P. Yao, and Z. Deng, "Multiwavelength actively mode-locked fiber ring laser with suppressed homogeneous line broadening and reduced supermode noise," Opt. Exp., vol. 12, pp. 4529-4534, Sep. 2004.

[12] B. Ortaç, M. Plötner, J. Limpert, and A. Tünnermann, "Self-starting passively mode-locked chirped pulse fiber laser," Opt. Exp., vol. 15, pp. 16794-16799, Dec. 2007.

[13] P. L. Urquhart, "Review of rare earth doped fibre lasers and amplifiers," Proc. Inst. Elect. Eng., vol. 135, pp. 385-407, Dec. 1988.

[14] O. Svelto, Principle of Lasers. New York: Springer, 1998.

[15] Z. Deng and J. P. Yao, "Photonic generation of microwave signal using a rational harmonic mode-locked fiber ring laser," IEEE Trans. Microw. Theory Tech., vol. 54, no. 2, pp. 763-767, Feb. 2006.

[16] J. E. Sharping, "Microstructure fiber based optical parametric oscillators," J. Lightw. Technol., vol. 26, no. 14, pp. 2184-2191, Jul. 2008.

[17] J. Li, Y. Liang, and K. K. Y. Wong, "Millimeter-wave UWB signal generation via frequency up-conversion using fiber optical parametric amplifier," IEEE Photon. Technol. Lett., vol. 21, no. 9, pp. 1172-1174, Sep. 2009.

[18] J. Li, B. P. P. Kuo, and K. K. Y. Wong, "Ultra-wideband pulse generation based on cross-gain modulation in fiber optical parametric amplifier," IEEE Photon. Technol. Lett., vol. 21, no. 2, pp. 212-214, Feb. 2009.

[19] M. E. Marhic, K. K.-Y. Wong, L. G. Kazovsky, and T.-E. Tsai, "Continuous-wave fiber optical parametric oscillator," Opt. Lett., vol. 27, pp. 1439-1441, 2002.

[20] M. E. Marhic, Fiber Optical Parametric Amplifiers, Oscillators and Related Devices. Cambridge, U.K.: Cambridge Univ. Press, 2008, ch. 12.

[21] S. Yang, Y. Zhou, J. Li, and K. K. Y. Wong, "Actively mode-locked fiber optical parametric oscillator," IEEE J. Sel. Topics Quantum Electron., vol. 15, no. 2, pp. 393-398, Mar. 2009.

[22] J. Lasri, P. Devgan, R. Tang, V. S. Grigoryan, W. L. Kath, and P. Kumar, "Regeneratively modelocked dual-wavelength soliton-pulse fibre-optical parametric oscillator in $C$ - and $L$-bands," Electron. Lett., vol. 40, 2004, Art. ID 20040425.

[23] C. C. Lee, Y. K. Chen, and S. K. Liaw, "Single-longitudinal-mode fiber laser with a passive multiple-ring cavity and its application for video transmission," Opt. Lett., vol. 23, pp. 358-360, 1998. 
[24] C. Yeh and S. Chi, "A broadband fiber ring laser technique with stable and tunable signal-frequency operation," Opt. Exp., vol. 13, pp. $5240-5244,2005$.

[25] K. Zhang and J. U. Kang, "C -band wavelength-swept single-longitudinal-mode erbium-doped fiber ring laser," Opt. Exp., vol. 16, pp. 14173-14179, 2008.

[26] S. Yang, X. Xu, Y. Zhou, K. K. Y. Cheung, and K. K. Y. Wong, "Continuous-wave single-longitudinal-mode fiber optical parametric oscillator with reduced pump threshold," IEEE Photon. Technol. Lett., vol. 21, no. 12, pp. 1870-1872, Dec. 2009.

[27] S. Yang, K. K. Y. Cheung, Y. Zhou, and K. K. Y. Wong, "Tunable single-longitudinal-mode fiber optical parametric oscillator," Opt. Lett. , accepted for publication.

[28] H. A. Haus, "Mode-locking of lasers," IEEE J. Sel. Topics Quantum Electron., vol. 6, no. 6, pp. 1173-1185, Nov. 2000.

[29] G. P. Agrawal, Nonlinear Fiber Optics, 4th ed. Boston, MA: Academic, 2007.

[30] G. P. Agrawal, Nonlinear Fiber Optics, 4th ed. Boston, MA: Academic, 2007, ch. 10.

[31] A. Gloag, R. J. Forster, and N. Langford, "Theoretical model and experimental demonstration of frequency control in rare-earth-doped fiber lasers with a $3 \times 3$ nonplanar fused-fiber coupler," J. Opt. Soc. Amer. B, Opt. Phys., vol. 14, pp. 985-901, 1997.

[32] Y. Han, O. Boyraz, and B. Jalali, "Tera-sample per second real-time waveform digitizer,” Appl. Phys. Lett., vol. 87, 2005, Art. ID 241116.

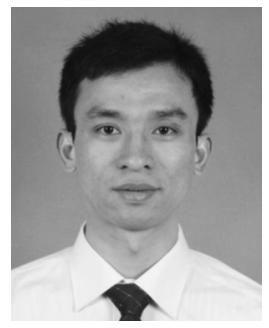

Sigang Yang received the B.E. degree in material from Northeastern University, Shenyang, China, in 2000, the M.S. degree in physical electronics from the Huazhong University of Science and Technology, Wuhan, China, in 2003, and the Ph.D. degree in electronic engineering from Tsinghua University, Beijing, China, in 2008.

$\mathrm{He}$ is currently with the Photonic Systems Research Laboratory, Department of Electrical and Electronic Engineering, The University of Hong Kong, Hong Kong. His past research interests included photonic crystal fibers and slow light. His current research interests include FOPA and oscillators.

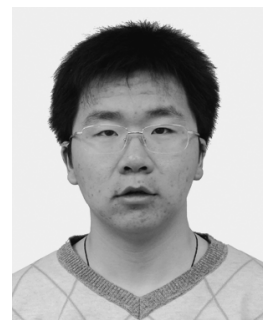

Chi Zhang received the B.S. degree in optical information science and technology from the Huazhong University of Science and Technology, Wuhan, China, in 2009, and is currently working toward the Ph.D. degree in the electrical and electronic engineering from the University of Hong Kong, Hong Kong.

His research interests include applications of fiber optical parametric AMPs, optical pulse generation, and signal processing.

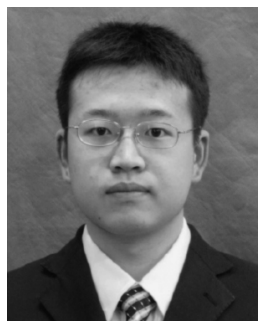

Yue Zhou received the B.Eng. degree in electronic engineering from Shanghai Jiao Tong University, Shanghai, China, in 2008, and s currently working toward the Ph.D. degree in electrical and electronic engineering at the University of Hong Kong, Hong Kong.

His current research interests are fiber optical parametric AMPs and oscillators.

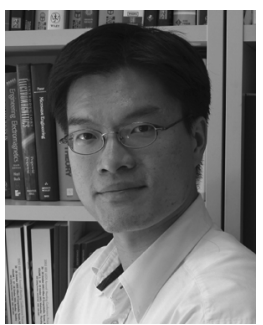

Kenneth Kin-Yip Wong (S'00-M'03) received the combined B.E. degree (first-class honors with a medal award) in electrical engineering and B.S. degree in physics from the University of Queensland, Brisbane, Qld., Australia, in 1997, and the M.S. and Ph.D. degrees from Stanford University, Stanford, CA, in 1998 and 2003, respectively, both in electrical engineering.

He was a member of the Photonics and Networking Research Laboratory, Stanford University. From 1998 to 1999, he was with Hewlett-Packard Laboratories, as a Research Engineer, during which time he contributed on projects that included parallel optics and vertical-cavity surface-emitting lasers (VCSELs). He was also an independent Consultant with Innovation CORE (a Sumitomo Electric Company, Yokohama, Japan), Santa Clara, CA, in 2004. $\mathrm{He}$ is currently an Assistant Professor with the Department of Electrical and Electronic Engineering, University of Hong Kong, Hong Kong. He has authored or coauthored over 50 journal and conference papers. He is the Reviewer for Optics Letters, the Journal of the Optical Society of America B, Optical Physics, Optics Express, IEE Electronics Letters, and Optics Communications. His past research interests included dense wavelength-division multiplexing (DWDM) systems, subcarrier multiplexed (SCM) optical systems, fiber nonlinearity, fiber optical parametric AMPs, and photonic crystal fibers.

Dr. Wong is a member of The International Society for Optical Engineers (SPIE) and the IEEE Lasers and Electro-Optic Society (LEOS). He is a reviewer for the IEEE PHOTONICS TECHNOLOGY LETTERS and the JOURNAL OF LightWAVE TECHNOLOGY. He was the recipient of the 2003 Optical Society of America (OSA) New Focus Student Award and the 2003 IEEE LEOS Graduate Student Fellowship. He was also the recipient of the 2005-2006 Best Teacher Award from the University of Hong Kong. 